\title{
EFECTO DEL USO DE SUELO EN LAS HORMIGAS (FORMICIDAE: HYMENOPTERA) DE TIKINMUL, CAMPECHE, MÉXICO
}

\author{
Cristina Isabel Chanatásig-V aCA, ${ }^{1 *}$ Esperanza Huerta Lwanga, ${ }^{2}$ \\ Patricia Rojas Fernández, ${ }^{3}$ Alejandro Ponce-Mendoza, ${ }^{2}$ Jorge Mendoza \\ Vega, ${ }^{1}$ Alejandro Morón Ríos, ${ }^{1}$ Hans VAN der WAL ${ }^{2}$ \& Benito Bernardo \\ Dzib-Castillo ${ }^{1}$ \\ ${ }^{1}$ El Colegio de la Frontera Sur, Calle 10 x 61 núm. 264, Col. Centro, CP 24000, San Francisco de \\ Campeche, Campeche, México.<jmendoza@ecosur.mx>, <amoron@ecosur.mx>, <bdzib@ecosur.mx> \\ ${ }^{2}$ El Colegio de la Frontera Sur, Carretera Villahermosa-Reforma km 15.5, Ranchería Guineo, sección \\ II CP 86280, Villahermosa, Tabasco, México.<ehuerta@ecosur.mx>, <Jano_ponce@hotmail.com>, \\ $<$ hvanderwal@ecosur.mx> \\ ${ }^{3}$ Instituto de Ecología, A.C., Km 2.5 Car. Antigua a Coatepec 351, Congregación El Haya, Xalapa \\ 91070, Veracruz, México. <patricia.rojas@inecol.edu.mx> \\ *Autor para correspondencia: <cchanata@ecosur.mx $><$ cchanatasig@yahoo.com.mx $>$
}

Chanatásig-Vaca, C. I., E. Huerta Lwanga, P. Rojas Fernández, A. Ponce-Mendoza, J. Mendoza Vega, A. Morón Ríos, H. Van der Wal \& B. B. Dzib-Castillo. 2011. Efecto del uso de suelo en las hormigas (Formicidae: Hymenoptera) de Tikinmul, Campeche, México. Acta Zoológica Mexicana (n. s.), 27(2): 441-461.

RESUMEN. Se comparó el efecto del uso de suelo sobre la mirmecofauna del ejido de Tikinmul, Campeche, México. El estudio se llevó a cabo en monocultivos de cedro y huertos caseros, durante la época seca y lluviosa. Para la colecta de especies se utilizó el método Tropical Soil Biology and Fertility (TSBF) modificado para sistemas agroforestales. Se encontraron un total de 37 morfoespecies, de las cuales nueve son exclusivas de cada uno de los dos sistemas, mientras 19 están presentes en ambos. Se observa una similitud (Sörensen) de 60.4\% entre sistemas y 68\% entre épocas. La subfamilia Myrmicinae con 8 géneros y 17 especies fue la mejor representada en el estudio. Solenopsis geminata y Dorymyrmex sp. fueron especies dominantes e indicadoras del monocultivo. La densidad de hormigas fue más alta en los monocultivos. No se encontró relación directa de la intensidad de manejo con la riqueza de especies. La cantidad de hojarasca se muestra como el factor determinante de abundancia de las hormigas. Los agroquímicos afectan de manera indirecta a sus poblaciones.

Palabras clave: agroecosistemas, riqueza de especies, mirmecofauna.

Chanatásig-Vaca, C. I., E. Huerta Lwanga, P. Rojas Fernández, A. Ponce-Mendoza, J. Mendoza Vega, A. Morón Ríos, H. Van der Wal \& B. B. Dzib-Castillo. 2011. Effect of soil use in ants

Recibido: 01/10/2010; aceptado: 11/03/2011. 
(Formicidae: Hymenoptera) in Tikinmul, Campeche, Mexico. Acta Zoológica Mexicana (n. s.), 27(2): 441-461.

ABSTRACT. The effect of land use on ants was compared in Tikinmul, Campeche, Mexico. The research was carried out in cedar monocultures and home gardens, during dry and wet seasons. TSBF method modified to agroforestry systems was used to collect ants. 37 species were collected, nine of which were exclusive to both systems, while 19 were present in both systems. A similarity (Sörensen index) of $60.4 \%$ between systems and $68 \%$ between seasons was observed. The Myrmecinae Subfamily with eight genera and 17 species was the most represented. Solenopsis geminata and Dorymyrmex sp. were dominant and indicator species of monoculture system. Ant density was higher in monocultures. A direct relationship between management intensity and species richness was not found. Litter amount appear to be the determinant factor to define ants abundance. Ants populations were affected indirectly by agrochemicals.

Key words: Agrosystems, species richness, mirmecofauna.

\section{INTRODUCCIÓN}

Los cambios en la composición de la vegetación (i.e. de sistemas diversos a monocultivos), aunados a la degradación física y química del suelo, pueden impactar en la abundancia y composición de especies (Brown et al. 2004, Lavelle et al. 2001, Wolters et al. 2000) y afectar las propiedades o atributos básicos de los ecosistemas (Masera et al. 1999) y los bienes y servicios que éstos proveen a la humanidad (Duffy 2002, Hooper et al. 2005).

Las propiedades del ecosistema dependen fuertemente de la biota, en términos de las características funcionales de los organismos presentes y la distribución y abundancia de estos organismos en el espacio y el tiempo (Brussard 1998). En este sentido las hormigas juegan un papel como ingenieros del ecosistema (Folgarait 1998, Jones et al. 1994) influenciando procesos biogeoquímicos que pueden afectar la disponibilidad de recursos, los flujos de materiales, las condiciones de humedad y temperatura del suelo, que afectan a otros organismos y procesos del sistema (Gutierrez \& Jones 2006, Huhta 2007, da Silva et al. 2009). Las hormigas además son bioindicadores de disturbio debido a su alta diversidad y abundancia, a la variedad de nichos que ocupan, a su rápida respuesta a cambios ambientales y a su identificación relativamente fácil, pudiendo ser útiles en la evaluación de respuestas bióticas frente a prácticas agrícolas como la fertilización, la fumigación y las quemas (Folgarait 1998, Graham et al. 2008, Peck et al. 1998).

En la Península de Yucatán los descendientes de la cultura Maya mantienen un rico acervo de prácticas agrícolas en sus agroecosistemas, entre ellas los huertos caseros (Montagnini 2006). Estos a menudo son considerados como estados intermedios entre un bosque natural y plantaciones forestales, debido a la alta diversidad de especies vegetales que se mantiene en ellos (Altieri 1991, Torquebiau \& Penot 2006). De acuerdo a Lok (1998), esta alta diversidad del agroecosistema contribuye al mantenimiento de los organismos del suelo. 
En el ejido de Tikinmul, los pobladores, en su mayoría de ascendencia maya, practican una agricultura tradicional en los huertos caseros que mantienen junto a sus viviendas, donde el laboreo consiste principalmente en el chapeo manual y donde el uso de agroquímicos es escaso. A la vez aplican una forma intensiva de agricultura en parcelas que son usadas con fines comerciales, en las cuales, al contrario del huerto casero, se utilizan frecuentemente productos químicos y métodos de laboreo mecánicos como la rastra. La conversión de un manejo agrícola tradicional a uno mecanizado puede tener secuelas en la biota del suelo, que pueden derivar en consecuencias negativas para el funcionamiento del agroecosistema. En el presente estudio se evaluaron los efectos de dos tipos de manejo agrícola en la mirmecofauna del suelo: manejo con bajos insumos en los huertos caseros, y manejo intensivo en plantaciones monoespecíficas de cedro (Cedrela odorata L.); teniendo como hipótesis que la intensificación en el manejo agrícola disminuye la diversidad y altera la composición y abundancia relativa de las especies de hormigas presentes en suelo.

\section{MATERIALES Y MÉTODOS}

Área de estudio. El poblado de Tikinmul pertenece al municipio de Campeche, México, se encuentra ubicado en las coordenadas $90^{\circ} 13^{\prime} 23^{\prime \prime}$ O y $19^{\circ} 45^{\prime} 55^{\prime \prime} \mathrm{N}$; a una altura promedio de $20 \mathrm{~m} \mathrm{snm}$. De acuerdo a la clasificación de Koeppen el clima es muy cálido subhúmedo (Aw1(w)(i’)) (Orellana 1999).

Diseño del muestreo. Se seleccionaron cinco predios en cada uno de los dos tipos de agroecosistemas estudiados: a) plantaciones forestales monoespecíficas de cedro y b) huertos caseros donde se encontraron además del cedro, especies frutales.

En cada sistema se tuvieron cinco repeticiones, que dieron un total de 10 unidades de muestreo. En el caso de las plantaciones de cedro el tamaño de la unidad de muestreo fue de $10,000 \mathrm{~m}^{2}$, mientras que en los huertos caseros la superficie fue de $2,500 \mathrm{~m}^{2}$. Para la selección de las parcelas de muestreo, se realizó un recorrido por el área de estudio, tanto en la zona de producción, como en la zona urbana donde se encuentran los huertos caseros. Se identificaron parcelas con características similares en cuanto a topografía, tipo de suelo, especies cultivadas y edad de las plantas de cedro ( 3 a 4 años). Adicionalmente se entrevistó a los propietarios para obtener información acerca del uso y manejo actual y anterior del suelo. Con esta información se seleccionaron las parcelas con características similares que constituyeron las repeticiones dentro de cada uno de los sistemas estudiados.

En cada parcela o unidad de muestreo se seleccionaron aleatoriamente cuatro sitios de muestreo o unidades representativas más pequeñas (URP) sensu Lavelle et al. (2003); compuestas cada una por un árbol de cedro y cuatro monolitos de suelo dispuestos en línea recta, con una separación de $50 \mathrm{~cm}$ entre ellos. Se realizaron dos 
muestreos en campo, uno durante la época seca (marzo 2008) y otro al final de la época de lluvia (diciembre 2008).

Muestreo en campo. En cada URP se extrajeron, con la ayuda de una pala, cuatro monolitos de suelo de $25 \times 25 \mathrm{~cm}$ de ancho y $30 \mathrm{~cm}$ de profundidad. De la superficie de cada uno de los monolitos se colectó la hojarasca, para determinar su peso seco. Se colocó el suelo de cada monolito en diferentes recipientes, separando las muestras correspondientes a los primeros $10 \mathrm{~cm}$ de profundidad, del suelo que se encontraba entre los 10 y los $30 \mathrm{~cm}$; del suelo de cada profundidad se tomaron muestras de 100 $\mathrm{g}$ para determinar materia orgánica, $\mathrm{pH}$ y textura. Se tomaron muestras de suelo con cilindros metálicos para determinar su densidad aparente y el porcentaje de humedad en las dos profundidades analizadas $(0-10$ y $10-30 \mathrm{~cm})$. La colecta de macroinvertebrados se basó en el método de Tropical Soil Biology and Fertility (TSBF) (Anderson \& Ingram 1994), modificado para sistemas agroforestales por Lavelle et al. (2003). Los datos de manejo del suelo se obtuvieron a partir de una entrevista realizada a cada uno de los productores en cada colecta.

Análisis de datos. Para la determinación de materia orgánica se utilizó el método de Walkley y Black, para el pH la solución 1:2 en agua, la textura se determinó mediante el método del hidrómetro de bouyuocos, la humedad relativa por gravimetría y la densidad aparente por el método TSBF. Los datos de los parámetros físico-químicos del suelo fueron analizados con la prueba no paramétrica de Wilcoxon para determinar si existían diferencias entre sistemas a diferentes profundidades.

Se identificaron y contabilizaron las morfoespecies de hormigas presentes en los dos agroecosistemas y en las dos épocas de colecta, para obtener la riqueza de especies como medida de la diversidad. Adicionalmente se realizó un análisis para estimar el número total de especies de hormigas que se podrían encontrar en los agroecosistemas de Tikinmul, para lo cual se utilizó el Sofware "Species accumulation" (CIMAT 2003), que selecciona el mejor modelo para un grupo de datos y el número total de especies de acuerdo a este modelo (Díaz-Francés \& Soberón 2005). También se utilizó el índice de Similitud de Sörensen para la comparación de la diversidad entre sistemas.

Como medida de abundancia se utilizó la densidad, la biomasa, la frecuencia de ocurrencia (FO) y la frecuencia relativa (FR). Para determinar si existían diferencias en la densidad y la biomasa de las hormigas, entre sistemas, épocas y diferentes profundidades del suelo se aplicó la prueba de Wilcoxon. La FO indica el porcentaje de parcelas en las que se encontró una especie determinada; mientras la FR muestra el porcentaje que representa una especie respecto al total de especies encontradas.

$$
\begin{aligned}
& F O=(n / N) 100 \\
& F R=(n / \Sigma n) 100
\end{aligned}
$$


Donde:

$\mathrm{N}=$ número de parcelas donde se muestreó

$\mathrm{n}=$ número de parcelas donde se encontró la especie

$\Sigma \mathrm{n}=$ sumatoria del número de parcelas donde se encontraron todas las especies

Tanto la FO, como la FR consideran la presencia o ausencia de una especie en cada sitio de captura (parcela), sin tomar en cuenta la cantidad de individuos, como estrategia para salvar el problema de la alta desviación estándar, común en muestreos de hormigas (Rojas 2009, com. pers.).

Para determinar especies indicadoras de cada sistema se utilizó el Valor Indicador de especies (IndVal) propuesto por Dufrêne \& Legendre (1997); un valor $p<0.05$ y un porcentaje mayor del $70 \%$ permiten considerar a una especie como indicadora del sitio o hábitat (McGeoch et al. 2002, van Rensburg et al.1999). Se utilizaron las funciones 'IndVal' para el cálculo del Ind Val y p-adjust para el valor p, ambas del programa "Labdsv" de la librería de R (http://cran.rproject.org/web/packages/labdsv/ index.html).

Se realizó un Análisis de Componentes Principales (ACP) a los datos físico-químicos del suelo, variables de manejo y de morfoespecies de hormigas. A partir del ACP se realizó un análisis de correlación canónica (ACC) (Ter Braak 1986) para detectar cuales de las variables físico-químicas y de manejo explican variaciones en la comunidad de hormigas. Se utilizó el paquete ADE-4 (Analysis of Ecological Data: Exploratory and Euclidean methods in Environmental sciences ) para el ACP (Chessel et al. 2004) y el ACC (Dray \& Dufour 2007). Para la ejecución de todos los programas estadísticos se utilizó el ambiente R 2.9 (R Development Core Team 2008).

\section{RESULTADOS}

Propiedades físico-químicas y de manejo del suelo. Los suelos en Tikinmul tienen una textura arcillosa (Cuadro 1). Sin embargo en los huertos caseros se presenta una tendencia hacia la textura franco-arcillosa. De esta manera los porcentajes de arcilla son significativamente mayores en los monocultivos $(p<0.001)$, mientras la arena y el limo son significativamente mayores $(p<0.001)$ en los huertos caseros. La biomasa de hojarasca es significativamente mayor en el monocultivo $(p<0.001)$. El suelo de los huertos caseros tiene mayores contenidos de materia orgánica $(p<0.001)$ en comparación con el suelo de los monocultivos. Mientras la densidad aparente del suelo es mayor en los monocultivos $(p<0.0001)$. Los suelos de ambos sistemas son ligeramente básicos, sin embargo el $\mathrm{pH}$ del huerto casero es significativamente mayor $(p<0.001)$. Los contenidos de humedad relativa variaron significativamente $(p<$ $0.0001)$ entre profundidades dentro de cada sistema. El mayor contenido de humedad 


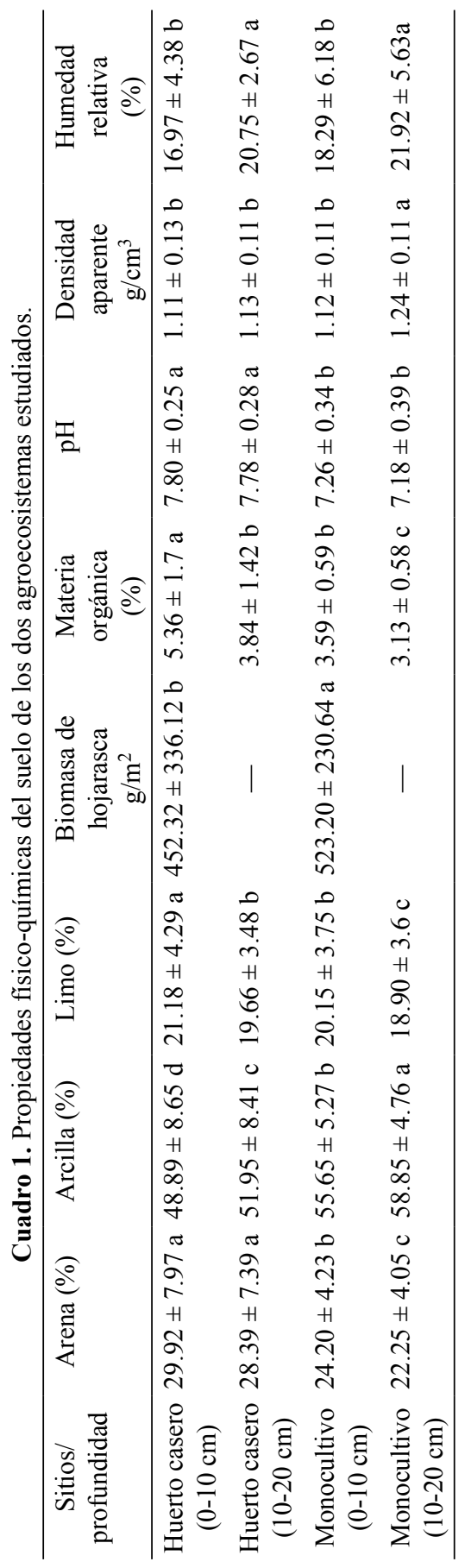


se encontró entre los 10-30 cm de profundidad del suelo de ambos sistemas. No hubo diferencia entre sistemas.

Las prácticas de manejo más comunes observadas en el ejido de Tikinmul, incluyen el uso de agroquímicos principalmente en el monocultivo, con el objetivo de fertilizar, y eliminar plagas y malezas, aunque estas últimas son controladas también con métodos mecánicos como el chapeo y el uso de la rastra. Los fertilizantes más utilizados son el Triple $17(17 \%$ N, 17\% P y $17 \% \mathrm{~K})$, el $18-46-00(18 \% \mathrm{~N}$ y $46 \% \mathrm{P})$ y la urea $(45 \% \mathrm{~N})$. Los herbicidas que tienen como principio activo el Glifosato (Nfosfonometil glicina) son de uso más difundido en la región. El riego es también una práctica común en el ejido y se realiza con la ayuda de una bomba que extrae agua de los mantos freáticos del suelo, para ser transportada por canales que llegan a cada una de las parcelas.

Diversidad y abundancia de hormigas. Se encontró un total de 37 especies, de las cuales nueve de ellas fueron exclusivas de cada uno de los dos sistemas, mientras 19 están presentes en huertos caseros como en monocultivos (Cuadro 2). La estimación del número total de especies que se podrían encontrar en los agroecosistemas de Tikinmul es de 52.

La subfamilia Myrmicinae con ocho géneros y 16 especies fue la mejor representada; seguida de las subfamilias Ecitoninae con dos géneros y tres especies y Formicinae junto a Dolichoderinae, también con dos géneros, pero con dos especies cada una. Pseudomyrmecinae, Proceratiinae, Ectatominae y Cerapachinae están representadas cada una por un género; de éstas la primera subfamilia tiene cuatro especies y las restantes una sola. Las hormigas Ponerinae están conformadas por cuatro géneros y siete especies.

Pheidole con cinco especies fue el género más rico, Hypoponera y Pseudomyrmex reúnen a cuatro especies, Solenopsis a tres, Crematogaster, Tetramorium, Cardiocondyla, Leptogenys, Labidus y Paratrechina a dos especies por género, mientras los demás géneros están representados por una sola.

El análisis de la diversidad beta (Indice de Sörensen) entre ambos sistemas indica que presentan un $60 \%$ de similitud, en cuanto que la similitud entre épocas es de $68 \%$.

Los datos de frecuencia de ocurrencia (FO), frecuencia relativa (FR) y el valor indicador de especies (Ind Val) (Cuadro 3) muestran la dominancia de S. geminata $y$ Dorymyrmex sp., que en conjunto representan más de la cuarta parte de observaciones en los monocultivos. En la FR se observa equidad en cuanto al porcentaje que representa cada una de las especies colectadas en los huertos caseros, respecto al total. Las especies del género Pheidole también tienen una alta presencia tanto en el huerto casero como en el monocultivo; en el huerto casero Pheidole sp. 4 representa más de la décima parte del total de individuos, y en conjunto, las especies de este género son casi la tercera parte de las hormigas de este sistema y la cuarta parte de las hormigas del monocultivo. 
Chanatásig-Vaca et al.: Hormigas en agroecosistemas de Tikinmul

Cuadro 2. Riqueza de especies de hormigas en los agroecosistemas de Tikinmul

\begin{tabular}{|c|c|c|c|}
\hline Subfamilia & Morfoespecie & Monocultivo & Huerto casero \\
\hline \multirow[t]{16}{*}{ Myrmicinae } & Pheidole sp 1 & $\mathrm{X}$ & $\mathrm{X}$ \\
\hline & Pheidole sp 2 & & $\mathrm{X}$ \\
\hline & Pheidole sp 3 & $X$ & $X$ \\
\hline & Pheidole sp 4 & $\mathrm{X}$ & $\mathrm{X}$ \\
\hline & Pheidole sp 5 & $\mathrm{X}$ & $\mathrm{X}$ \\
\hline & Solenopsis geminata & $\mathrm{X}$ & $\mathrm{X}$ \\
\hline & Solenopsis sp 1 & & $\mathrm{X}$ \\
\hline & Solenopsis sp 2 & $\mathrm{X}$ & \\
\hline & Cardiocondyla nuda & & $\mathrm{X}$ \\
\hline & Cardiocondyla reina & $\mathrm{X}$ & \\
\hline & Crematogaster sp 1 & & $\mathrm{X}$ \\
\hline & Crematogaster sp 2 & $\mathrm{X}$ & $\mathrm{X}$ \\
\hline & Tetramorium similimun & & $\mathrm{X}$ \\
\hline & Tetramorium spinosun & & $\mathrm{X}$ \\
\hline & Acromyrmex octospinosus & $\mathrm{X}$ & \\
\hline & Cephalotes minutus & & $\mathrm{X}$ \\
\hline \multirow[t]{7}{*}{ Ponerinae } & Hypoponera sp 1 & $\mathrm{X}$ & $X$ \\
\hline & Hypoponera sp 3 & $\mathrm{X}$ & $X$ \\
\hline & Hypoponera nitidula & $\mathrm{X}$ & \\
\hline & Leptogenys sp 1 & $\mathrm{X}$ & $\mathrm{X}$ \\
\hline & Leptogenys sp 2 & & $\mathrm{X}$ \\
\hline & Odontomachus yucatecus & $\mathrm{X}$ & $\mathrm{X}$ \\
\hline & Pachycondyla sp & $\mathrm{X}$ & $\mathrm{X}$ \\
\hline \multirow[t]{4}{*}{ Pseudomyrmecinae } & Pseudomyrmex sp 1 & $\mathrm{X}$ & $\mathrm{X}$ \\
\hline & Pseudomyrmex sp 2 & $\mathrm{X}$ & $\mathrm{X}$ \\
\hline & Pseudomyrmex sp 3 & $\mathrm{X}$ & \\
\hline & Pseudomyrmex sp 4 & $\mathrm{X}$ & \\
\hline \multirow[t]{3}{*}{ Ecitoninae } & Labidus coecus & $\mathrm{X}$ & $\mathrm{X}$ \\
\hline & Labidus predator & $\mathrm{X}$ & $\mathrm{X}$ \\
\hline & Nomamyrmex sp & & $\mathrm{X}$ \\
\hline \multirow[t]{2}{*}{ Formicinae } & Camponotus sp & $\mathrm{X}$ & \\
\hline & Paratrechina longicornis & $\mathrm{X}$ & $\mathrm{X}$ \\
\hline \multirow[t]{2}{*}{ Dolichoderinae } & Dorymyrmex sp & $\mathrm{X}$ & $\mathrm{X}$ \\
\hline & Forelius sp & $\mathrm{X}$ & $\mathrm{X}$ \\
\hline Proceratiinae & Proceratium $\mathrm{sp}$ & $\mathrm{X}$ & \\
\hline Ectatomminae & Ectatomma tuberculatum & $\mathrm{X}$ & \\
\hline Cerapachinae & Acanthostichus sp & $\mathrm{X}$ & $\mathrm{X}$ \\
\hline Total de especies & 37 & 28 & 28 \\
\hline
\end{tabular}


Cuadro 3. Frecuencia de ocurrencia (FO), Frecuencia relativa (FR) y Valor indicador (Ind Val) de las especies de hormigas presentes en agroecosistemas de Tikinmul, Campeche. Un Ind Val igual o mayor del $70 \%$ y $\mathrm{p}<0.05$ califican a una especie como indicadora.

\begin{tabular}{|c|c|c|c|c|c|c|c|c|}
\hline \multirow[b]{2}{*}{ Morfoespecies } & \multicolumn{4}{|c|}{ Huertos caseros } & \multicolumn{4}{|c|}{ Monocultivos de cedro } \\
\hline & FO & FR & $\begin{array}{c}\text { Ind Val } \\
\%\end{array}$ & $\mathrm{p}$ & FO & FR & $\begin{array}{c}\text { Ind Val } \\
\%\end{array}$ & $\mathrm{p}$ \\
\hline Solenopsis geminata & 60.0 & 9.4 & 2.8 & $\mathrm{a}$ & 100.0 & 13.0 & 85.9 & $\mathrm{a}$ \\
\hline Dorymyrmex $\mathrm{sp}$ & 50.0 & 7.8 & 4.5 & $\mathrm{a}$ & 90.0 & 11.7 & 81.9 & $\mathrm{a}$ \\
\hline Pheidole sp 4 & 50.0 & 7.8 & 23.2 & $\mathrm{a}$ & 60.0 & 7.8 & 32.1 & $\mathrm{~b}$ \\
\hline Leptogenys sp 1 & 40.0 & 6.3 & 28.8 & $\mathrm{a}$ & 50.0 & 6.5 & 14.0 & $\mathrm{~b}$ \\
\hline Labidus coecus & 40.0 & 6.3 & 1.6 & $\mathrm{a}$ & 50.0 & 6.5 & 48.0 & $\mathrm{~b}$ \\
\hline Pheidole sp 1 & 30.0 & 4.7 & 8.4 & $\mathrm{a}$ & 50.0 & 6.5 & 36.0 & $\mathrm{~b}$ \\
\hline Acanthostichus sp & 20.0 & 3.1 & 11.1 & $\mathrm{a}$ & 40.0 & 5.2 & 17.8 & $\mathrm{~b}$ \\
\hline Pheidole sp 5 & 50.0 & 7.8 & 48.8 & $\mathrm{a}$ & 30.0 & 3.9 & 1.2 & $\mathrm{~b}$ \\
\hline Solenopsis $\mathrm{sp} 1$ & 0.0 & 0.0 & 0.0 & $\mathrm{a}$ & 30.0 & 3.9 & 30.0 & $\mathrm{~b}$ \\
\hline Pseudomyrmex sp 1 & 0.0 & 0.0 & 0.0 & $\mathrm{a}$ & 30.0 & 3.9 & 30.0 & $\mathrm{~b}$ \\
\hline Pseudomyrmex sp 4 & 0.0 & 0.0 & 0.0 & $\mathrm{a}$ & 30.0 & 3.9 & 30.0 & $\mathrm{~b}$ \\
\hline Hypoponera sp 3 & 30.0 & 4.7 & 26.0 & $\mathrm{a}$ & 20.0 & 2.6 & 2.7 & $\mathrm{~b}$ \\
\hline Odontomachus yucatecos & 20.0 & 3.1 & 10.0 & $\mathrm{a}$ & 20.0 & 2.6 & 10.0 & $\mathrm{~b}$ \\
\hline Hypoponera nitidula & 0.0 & 0.0 & 0.0 & $\mathrm{a}$ & 20.0 & 2.6 & 20.0 & $\mathrm{~b}$ \\
\hline Proceratium sp & 0.0 & 0.0 & 0.0 & $\mathrm{a}$ & 20.0 & 2.6 & 20.0 & $\mathrm{~b}$ \\
\hline Ectatomma tuberculatum & 0.0 & 0.0 & 0.0 & $\mathrm{a}$ & 20.0 & 2.6 & 20.0 & $\mathrm{~b}$ \\
\hline Forelius sp & 30.0 & 4.7 & 14.4 & $\mathrm{a}$ & 10.0 & 1.3 & 10.4 & $\mathrm{~b}$ \\
\hline Paratrechina longicornis & 30.0 & 4.7 & 28.5 & $\mathrm{a}$ & 10.0 & 1.3 & 0.5 & $\mathrm{~b}$ \\
\hline Pheidole sp 3 & 10.0 & 1.6 & 6.7 & $\mathrm{a}$ & 10.0 & 1.3 & 3.3 & $\mathrm{~b}$ \\
\hline Pachycondyla sp & 10.0 & 1.6 & 3.3 & $\mathrm{a}$ & 10.0 & 1.3 & 6.7 & $\mathrm{~b}$ \\
\hline Pseudomyrmex sp 2 & 10.0 & 1.6 & 5.0 & $\mathrm{a}$ & 10.0 & 1.3 & 5.0 & $\mathrm{~b}$ \\
\hline Labidus predator & 10.0 & 1.6 & 1.4 & $\mathrm{a}$ & 10.0 & 1.3 & 8.6 & $\mathrm{~b}$ \\
\hline Crematogaster sp 2 & 0.0 & 0.0 & 0.0 & $\mathrm{a}$ & 10.0 & 1.3 & 10.0 & $\mathrm{~b}$ \\
\hline Acromyrmex octospinosus & 0.0 & 0.0 & 0.0 & $\mathrm{a}$ & 10.0 & 1.3 & 10.0 & $\mathrm{~b}$ \\
\hline Cardiocondyla reina & 0.0 & 0.0 & 0.0 & $\mathrm{a}$ & 10.0 & 1.3 & 10.0 & $\mathrm{~b}$ \\
\hline Pseudomyrmex sp 3 & 0.0 & 0.0 & 0.0 & $\mathrm{a}$ & 10.0 & 1.3 & 10.0 & $\mathrm{~b}$ \\
\hline Camponotus sp & 0.0 & 0.0 & 0.0 & $\mathrm{a}$ & 10.0 & 1.3 & 10.0 & $\mathrm{~b}$ \\
\hline Nomamyrmex sp & 30.0 & 4.7 & 30.0 & $\mathrm{a}$ & 0.0 & 0.0 & 0.0 & $\mathrm{~b}$ \\
\hline Crematogaster sp 1 & 20.0 & 3.1 & 20.0 & $\mathrm{a}$ & 0.0 & 0.0 & 0.0 & $\mathrm{~b}$ \\
\hline Pheidole sp 2 & 20.0 & 3.1 & 20.0 & $\mathrm{a}$ & 0.0 & 0.0 & 0.0 & $\mathrm{~b}$ \\
\hline Tetramorium spinosun & 20.0 & 3.1 & 20.0 & $\mathrm{a}$ & 0.0 & 0.0 & 0.0 & $\mathrm{~b}$ \\
\hline Solenopsis sp 2 & 10.0 & 1.6 & 10.0 & $\mathrm{a}$ & 0.0 & 0.0 & 0.0 & $\mathrm{~b}$ \\
\hline Cephalotes minutus & 10.0 & 1.6 & 10.0 & $\mathrm{a}$ & 0.0 & 0.0 & 0.0 & $\mathrm{~b}$ \\
\hline Tetramorium similimun & 10.0 & 1.6 & 10.0 & $\mathrm{a}$ & 0.0 & 0.0 & 0.0 & $\mathrm{~b}$ \\
\hline Cardiocondyla nuda & 10.0 & 1.6 & 10.0 & $\mathrm{a}$ & 0.0 & 0.0 & 0.0 & $\mathrm{~b}$ \\
\hline Hypoponera sp 1 & 10.0 & 1.6 & 10.0 & $\mathrm{a}$ & 0.0 & 0.0 & 0.0 & $\mathrm{~b}$ \\
\hline Leptogenys sp 2 & 10.0 & 1.6 & 10.0 & $\mathrm{a}$ & 0.0 & 0.0 & 0.0 & $\mathrm{~b}$ \\
\hline
\end{tabular}


La alta FO aunado a un IndVal $>70 \%$ y una $p<0.05$ de $S$. geminata $(\mathrm{FO}=100$; $p=0.015)$ y Dorymyrmex sp. $(\mathrm{FO}=90 ; p=0.014)$ en los monocultivos, permiten considerarlas como especies indicadoras de este ecosistema; no obstante, S. geminata también tiene una alta representatividad en huertos caseros, debido a su FO de $59 \%$, que sólo se encuentra por debajo de Pheidole sp. 4. La FO mayor al 70\% en las dos especies indicadoras del monocultivo, evidencia además un patrón de dominancia en este sistema, que no ocurre con ninguna especie en los huertos caseros, donde se observa una comunidad más proporcionada con un índice de equidad de 0.3 , frente a 0.19 de los monocultivos.

La densidad de hormigas (Fig. 1) es significativamente mayor ( $p=0.0104)$ en los monocultivos $\left(121.85 \pm 104.14\right.$ individuos $\left./ \mathrm{m}^{2}\right)$ que en los huertos caseros $(51.70 \pm$ 70.57 individuos $/ \mathrm{m}^{2}$ ). Por otro lado, dentro de cada uno de los sistemas no existen diferencias estadísticamente significativas en la densidad entre las dos épocas ni a diferentes profundidades.

Correlación entre variables biológicas, físico-químicas y de manejo del suelo. Los dos ejes principales del ACP correspondiente a las características físico-químicas del suelo (Fig. 2a) explican el $62 \%(\mathrm{X}=42.8 ; \mathrm{Y}=19.2)$ de la variabilidad. De todas las variables físico-químicas estudiadas, solamente la arcilla y la densidad aparente están fuertemente relacionadas con los monocultivos, por presentar valores más elevados en este sistema. La arena, el limo, el $\mathrm{pH}$ y principalmente la materia orgánica tienen una mayor correspondencia con el sistema huerto casero. Existe una ligera diferenciación de las variables analizadas entre época seca y fin de lluvias.

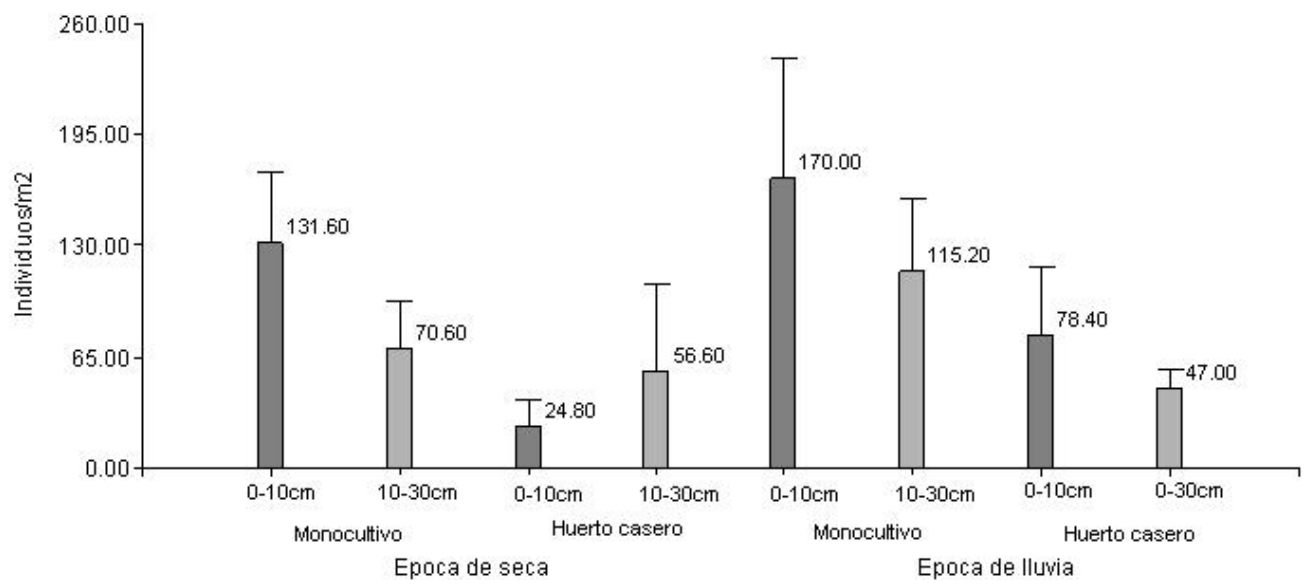

Figura 1. Densidad de hormigas en los dos agroecosistemas en época seca y lluviosa. 


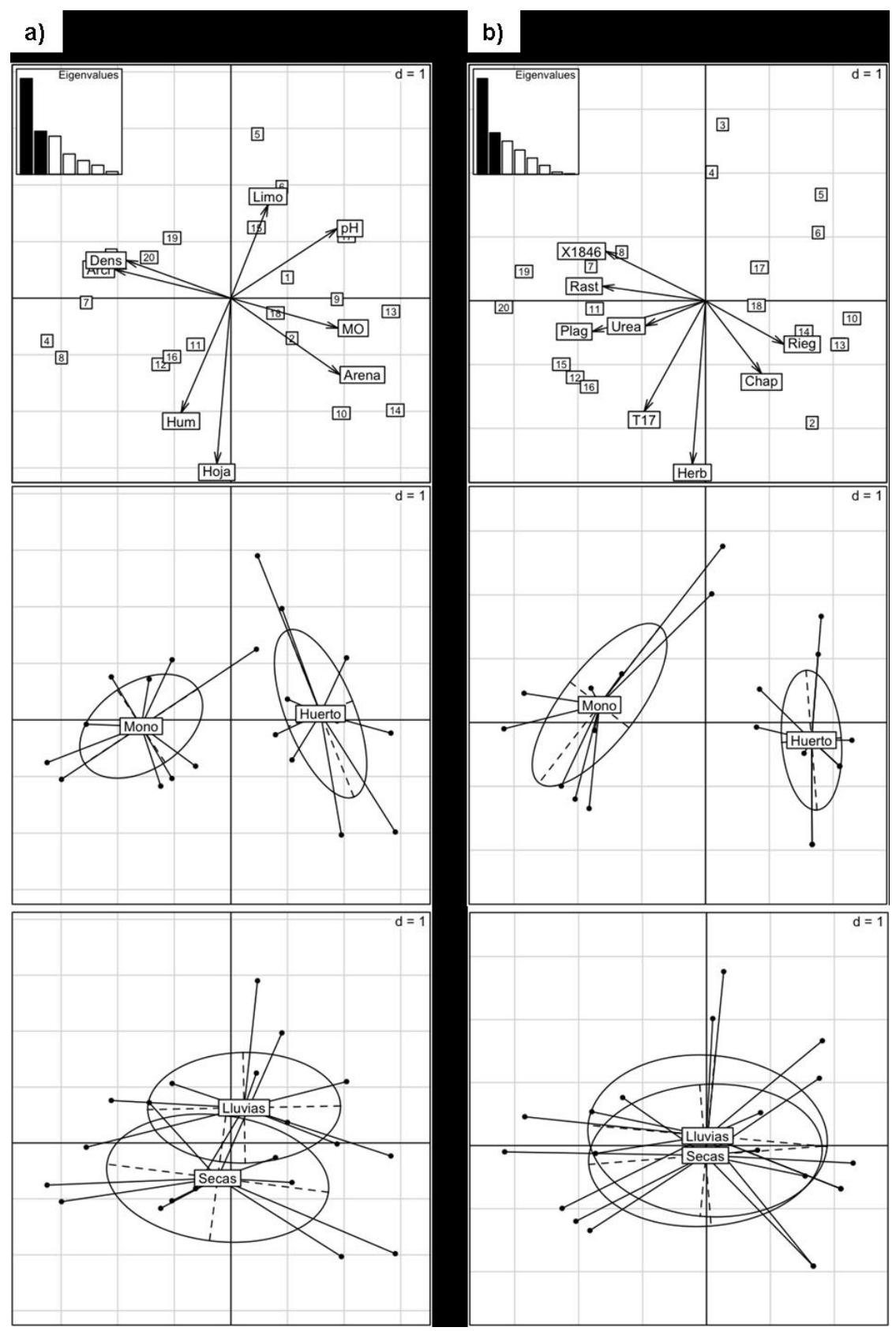

Figura 2. a) Análisis de Componentes Principales de las características físico-químicas del suelo; b) Análisis de Componentes principales de las características de manejo de las parcelas. 
En el ACP del manejo del suelo (Fig. 2b), los dos factores principales explican el $61.44 \%(X=42.84 \% ; \mathrm{Y}=18.60 \%)$ de la variabilidad y revelan que el manejo intensivo del suelo que implica la aplicación de productos químicos y métodos mecánicos de deshierbe, tiene mayor incidencia en el monocultivo. En los dos ACP del suelo, el eje vertical separa claramente los huertos caseros de los monocultivos y los distingue como dos sistemas diferentes con características físico-químicas y de manejo propias.

Al igual que el análisis de valor indicador de especies (Ind Val), los análisis de correlación canónica realizados con los datos de la abundancia de morfoespecies y las variables físico-químicas (Fig. 3a) y entre los mismos datos de las morfoespecies y las prácticas de manejo de suelo (Fig. 3b), indican la relevancia de $S$. geminata en los monocultivos. De acuerdo a los resultados los individuos de $S$. geminata se encuentran en donde se presentan valores más altos de densidad del suelo y se han realizado aplicaciones más recientes de plaguicidas, así como del empleo de la rastra. También Cardiocondyla sp., Pseudomyrmex sp. 4, Dorymyrmex sp. y Proceratium sp., se encuentran en el monocultivo asociadas a las mismas condiciones físico-químicas. Cuatro especies tienen una mayor afinidad con el huerto casero dada por las características físico-químicas del suelo, de esta manera Leptogenys sp. 1 se relaciona positivamente con el limo; Pseudomyrmex sp. 3 con la materia orgánica; Crematogaster sp. 2 y Acromyrmex octospinosum con el pH. Por otro lado, Solenopis sp. 1 y Solenopis sp. 2, Cephalotes minutus, Paratrechina longicornis, Hypoponera nitidula y Leptogenys sp. 1 presentan una correlación positiva con el fertilizante "Triple 17".

Tanto $S$. geminata, como Cardiocondyla reina, se encuentran en parcelas donde se aplicó insecticida y la densidad aparente del suelo es alta. Aunque $S$. geminata y Dorymyrmex sp. se encuentran preferentemente en el monocultivo, éstas habitan espacios con diferentes características, donde $S$. geminata se halla en áreas de mayor intensidad de manejo, mientras Dorymyrmex sp. se encuentra en áreas de menor intensidad. Ninguna de las especies de hormigas mostró asociación con la aplicación de herbicida.

\section{DISCUSIÓN}

La riqueza de especies conocida en Campeche antes del presente trabajo fue de 78 especies de hormigas (Chan 2002). En este estudio se encontraron 37 especies, de las cuales 17 son nuevos registros para el estado, con lo cual la riqueza de especie de Campeche se eleva a 95; superando así a Chiapas que registra 68 especies y quedando sólo atrás de Baja California con 170, Veracruz con 157 y Tabasco con 138, que son los estados con mayor número de especies reportadas de este grupo (Del Toro et al. 2009, Johnson \&Ward 2002).

Al igual que en esta comunidad, otros estudios realizados en México, reportan que la subfamilia con mayor número de géneros y especies es Myrmicinae, seguida del 


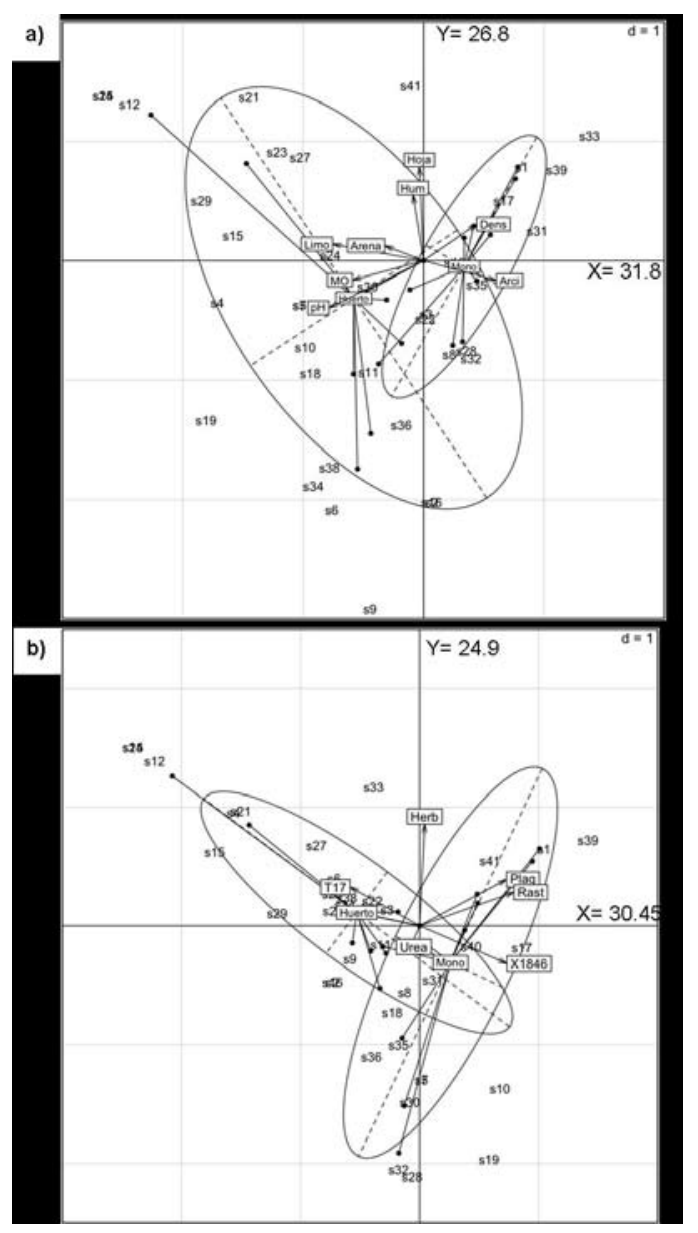

Figura 3. Correlación canónica entre morfoespecies de hormigas y a).características físico-químicas del suelo; y b). prácticas de manejo del suelo. S1: Solenopsis geminata; S2: Solenopsis sp 2; S3:

Solenopsis sp 1; S4: Crematogaster sp 1; S5:Crematogaster sp 2; S6:Cephalotes minutos; S7: Acromyrmes octospinosus; S8: Pheidole sp 1; S9: Pheidole sp 2; S10: Pheidole sp 3; S11: Pheidole sp 4; S12: Pheidole sp 5; S14: Tetramorium similimun; S15: Tetramorium spinosum; S16: Cardiocondyla nuda; S17: Cardiocondyla reina; S18: Pachicondila sp; S19: Hypoponera sp 1; S21: Hypoponera sp 3; S22: Hypoponera nitidula; S23: Odontomachus yucatecos; S24: Leptogenys sp 1; S25: Leptogenys sp 2; S27: Acanthostichus sp; S28: Pseudomyrmex sp 1; S29: Pseudomyrmex sp 2; S30: Pseudomyrmex sp 3; S31: Pseudomyrmex sp 4; S32: Labidus coecus; S33: Labidus predator; S34: Nomamyrmex sp; S35: Dorymyrmex sp; S36: Forelius sp; S38: Paratrechina longicornis; S39: Camponotus sp; S40: Proceratium sp; S41: Ectatomma tuberculatum; Hoja: biomasa de hojarasca; Hum: humedad relativa del suelo; MO: porcentaje de materia orgánica del suelo; Arci: porcentaje de arcilla; Arena: porcentaje de arena; Limo: porcentaje de limo; Fura: aplicación de herbicida furarán; Glifo:aplicación de herbicida glifosato; 1846: aplicación de fertilizante 18-46-0; Urea: aplicación de urea. 
grupo de las Ponerinae (Del Toro et al. 2009, Quiroz-Robledo \& Valenzuela-González 2007). Esta situación es común en áreas tropicales de acuerdo a Rojas (2001).

La riqueza de especies en diferentes trabajos se relaciona de manera positiva con la complejidad vegetal que a la vez determina una mayor cantidad de microhábitats (Rivas \& Schoereder 2007) y de recursos disponibles (Blüthgen et al. 2000), entre ellos la hojarasca (Kaspari et al. 2008); y de manera negativa con la intensidad de manejo (Philpott \& Armbrecht 2006). Sin embargo, en el presente trabajo no se encontró relación de la riqueza de especies de hormigas con la complejidad vegetal, ya que a pesar de que los monocultivos son sistemas menos complejos, albergan una riqueza de especies de hormigas similar a la de los huertos caseros; no obstante la composición de especies de hormigas es diferente para cada agroecosistema. No se encontró elementos suficientes para determinar que característica de los dos sistemas es determinante para la riqueza de especies.

La composición de las comunidades de hormigas de Tikinmul se ajusta a los patrones encontrados en el trópico americano y descritos por Fowler (1993), dentro de los cuales operan las condiciones específicas de los dos agroecosistemas estudiados, principalmente el microclima. De acuerdo a estos patrones, donde se presenta una dominancia del género Pheidole, también existe una alta representatividad de otros géneros de las subfamilias Mymicinae, de las Ponerinae y Pseudomyrmecinae $\mathrm{y}$ en contraste una baja presencia de los géneros de las subfamilias Formicinae y Ecitoninae; mientras para la subfamilia Dolichoderinae, los patrones son difusos, ya que aunque no es frecuente un desplazamiento al encontrarse presentes especies de Pheidole, eventualmente puede ocurrir (Fowler 1993, Lieberburg et al. 1975). En cuanto al microclima muchas especies de hormigas tropicales han mostrado ser sensibles a factores ambientales que pueden incrementar el riesgo de desecación, como la insolación y la baja humedad (Lighton \& Feener 1989, Kaspari \& Weiser 2000), aunque también existen especies que presentan predilección por los hábitats abiertos y expuestos al sol (MacGown \& Lockley 2009, Risch \& Carroll 1982, Torres-Contreras \& Vásquez 2004). De acuerdo a sus preferencias las especies de hormigas pueden inclinarse por hábitats más abiertos como el monocultivo (i.e. S. geminata y Dorymyrmex sp.), o con mayor cobertura vegetal como los huertos caseros. Al presentarse niveles de humedad del suelo similares en ambos agroecosistemas, no se pudo determinar la influencia de esta variable sobre las hormigas.

La hojarasca influye sobre las poblaciones de artrópodos del suelo (Sayer 2006) mediante la regulación del microclima (Bradford et al. 2002, González \& Seatedt 2001, Vasconcelos \& Laurance 2005) y la disponibilidad de nutrimentos ((Sayer 2006, Evetts 2008). En estudios realizados en zonas del trópico americano, en Brasil, Colombia y México la densidad de las poblaciones de hormigas se presenta muy variable e independiente del uso de suelo. De esta manera, es posible encontrar medias de densidades bajas de 11 individuos $/ \mathrm{m}^{2}$ (Decaëns et al. 1994) y altas de 603 (Brown 
et al. 2004) y hasta casi 3000 individuos $/ \mathrm{m}^{2}$ (Brown et al. 2001) en sistemas similares de cultivos de gramíneas (pasto, arroz y caña de azúcar). No obstante, en los sistemas donde se encontró mayor densidad poblacional de hormigas se reportó una alta cantidad de materia vegetal en el suelo (Barros et al. 2002, Brown et al. 2001). A este respecto, Kaspari y Yanoviak (2009) observaron una alta correlación entre el espesor de la hojarasca del suelo y la cantidad de hormigas en ecosistemas del trópico panameño y peruano, en donde, a un espesor de la hojarasca de entre $0.1 \mathrm{a} 1 \mathrm{~cm}$ correspondió una densidad de alrededor de 200 individuos $/ \mathrm{m}^{2}$, que aumentó proporcionalmente con el incremento de hojarasca, llegando a encontrarse más de 2000 individuos $/ \mathrm{m}^{2}$ donde la altura de la hojarasca superaba $\operatorname{los} 10 \mathrm{~cm}$. Estos resultados son congruentes con lo encontrado en el presente trabajo, ya que a un bajo espesor de la hojarasca que no superó los $3 \mathrm{~cm}$ correspondió una baja densidad poblacional de las hormigas no mayor a los 200 individuos por $\mathrm{m}^{2}$.

La alta densidad poblacional de $S$. geminata en ambos agroecosistemas, está dada por una buena estrategia de abastecimiento de recursos alimenticios y su adaptación a las características microclimáticas del sistema, principalmente en los monocultivos. Esta especie por sus hábitos generalistas y omnívoros (Carroll \& Janzen 1973, Levings 1983), puede disponer de un amplio rango de recursos mediante la colecta de semillas de maleza, materia orgánica de la vegetación que cae al suelo, así como de pequeños organismos sedentarios o de movimientos lentos, tales como huevos de insectos, larvas, pupas y algunos artrópodos adultos (Carroll \& Risch 1984, Perfecto 1994, Perfecto \& Sediles 1992, Risch 1980, Wilson 1978), que a la vez le dan mayores opciones de abastecimiento de alimento y le permiten competir con mucho éxito con otras especies del suelo (Rojas 2001).

La mayor abundancia de $S$. geminata, que resalta en su dominancia en los monocultivos, se explica por su preferencia por áreas abiertas (Risch \& Carroll 1982) e hidroquinesis negativa (Potts et al. 1983, Cokendolpher \& Francke 1985), dos condiciones similares a las encontradas en este sistema, donde los árboles están sembrados en espaciamientos de $3 \times 4 \mathrm{~m}$ y la humedad relativa del suelo es baja. Sin embargo, esta especie no persiste si se da paso a la sucesión vegetal (Risch \& Carroll 1982), posiblemente por el incremento de cobertura arbórea como consecuencia de este proceso. Lo que podría explicar la menor abundancia de esta hormiga en los huertos caseros, donde la vegetación genera una mayor cobertura vegetal a lo largo del año.

Las especies del género Dorymyrmex sp. también habitan lugares abiertos (MacGown \& Lockley 2009, Torres-Contreras \& Vásquez 2004) al igual que S. geminata. Posiblemente esta preferencia hace que ambas especies sean dominantes en el monocultivo; aunque no compartan el mismo espacio dentro de estos sistemas, ya que mientras $S$. geminata habita parcelas con manejo más intensivo, donde inclusive se han realizado aplicaciones de insecticidas, Dorymyrmex sp. se encuentra en mayor 
cantidad en parcelas con menor intensidad de manejo. Esto permite presumir que el éxito de $S$. geminata se basa en su tolerancia a los componentes del manejo intensivo como el uso de agroquímicos y la alta densidad aparente del suelo, en combinación con su eficiente estrategia de competencia por recursos. En otros agroecosistemas americanos también se han observado relaciones similares de dominancia compartida de $S$. geminata que puede converger en un mismo agroecosistema con Pheidole radoszkowskii sin competir por los recursos, debido a las diferencias en su comportamiento forrajero (Perfecto 1994).

A pesar de la tolerancia mostrada por $S$. geminata a los insecticidas, esta especie al igual que las otras hormigas, se muestra afectada por los herbicidas. La toxicidad directa de los herbicidas sobre los invertebrados del suelo parece ser baja (Bohan et al. 2005, Peterson \& Hulting 2004, Römbke \& García 2000), por lo que el efecto del glifosato sobre las poblaciones de hormigas puede ser una consecuencia secundaria a la pérdida de vegetación (Guiseppe et al. 2006, Guynn et al. 2004). Se puede hipotetizar que de la misma forma que los herbicidas afectan de manera indirecta las poblaciones de hormigas al eliminar la vegetación, los fertilizantes podrían beneficiar a la mirmecofauna al favorecer el crecimiento de las plantas.

De acuerdo a Crain y Bertness (2006) en ambientes bajo condiciones extremas, pequeñas alteraciones en los parámetros físicos podrían crear hábitats hospitalarios para organismos que de otra manera no serían capaces de tolerar condiciones físicas limitantes; mientras por otra parte en ambientes más benignos, pequeñas modificaciones al ambiente físico, pueden ser irrelevantes o inclusive reducir el hábitat apto para otras especies. De esta manera, la actividad de establecimiento de los nidos de $S$. geminata, que pueden llegar hasta $1.5 \mathrm{~m}$ de profundidad (Perfecto \& Vandermeer 1996), podría resultar beneficiosa tanto para la diversidad de la micro y macro fauna del suelo, como para los procesos biogeoquímicos del suelo, ya que mediante la remoción de suelo, se pueden afectar las condiciones abióticas como temperatura, humedad, disponibilidad de oxígeno y flujo de materiales que pueden a su vez promover el desarrollo de microorganismos que favorecen procesos como el ciclo del nitrógeno y del carbono (Gutierrez \& Jones 2006).

\section{CONCLUSIONES}

No se encontró relación de la intensidad de manejo agrícola con la riqueza de especies de hormigas. Por lo tanto se rechaza la hipótesis que indica que la intensificación en el manejo agrícola disminuye la diversidad y el número de especies de hormigas presentes en suelo. Sin embargo, sí se encontraron diferencias en la composición de especies.

Los sistemas se diferencian por la presencia de especies dominantes en los monocultivos, que no se presenta en los huertos caseros. Las dos especies dominantes 
de los monocultivos, $S$. geminata y Dorymyrmex sp. a la vez se caracterizan por ser especies indicadoras del monocultivo.

La influencia de los productos químicos como herbicidas y abonos sobre las poblaciones de hormigas, al parecer no es directa, más bien su incremento o decremento podrían ser una consecuencia secundaria de la acción directa de estos productos sobre la vegetación; sin embargo, es necesario realizar más estudios al respecto que corroboren estos resultados.

AGRADECIMIENTOS. Agradecemos al Gobierno del estado de Tabasco y al CONACYT, quienes a través del proyecto TaB-2007-C09-74864 de Fondos mixtos Tabasco, financiaron los gastos de campo y análisis de suelo; a los pobladores del ejido de Tikinmul, en particular a los productores que amablemente me permitieron realizar los muestreos en sus parcelas; de manera muy especial a los estudiantes del Instituto Tecnológico de Chiná, al personal de los laboratorios de Suelos de la Unidad ECOSURVillahermosa, de Vida Silvestre y Colecciones Científicas del CEDESU de la Universidad Autónoma de Campeche, y de Biología de suelos del Instituto de Ecología, A. C., con sede en Xalapa, por su valiosa colaboración en el trabajo de campo y de laboratorio y a Jorge Albino Vargas por sus oportunos consejos. A la memoria de Rosita Chan, quien realizó importantes contribuciones al conocimiento de la mirmecofauna en Campeche.

\section{LITERATURA CITADA}

Alonso, L. E. \& D. Agosti. 2000. Biodiversity studies, monitoring of ants: An Overview. pp.1-8. In: D. Agosti, J. D. Majer, L. E. Alonso \& T. R. Schultz (Eds.). Ants: Standard methods for measuring and monitoring biodiversity. Smithsonian Institution Press, Washington.

Altieri, M. 1991. Tradicional farming in Latin America. The Ecologist, 21: 93-96.

Andersen, A.N. 1990. The use of ant communities to evaluate change in Australian terrestrial ecosystem: A review and recipe. Proceedings of the ecological Society of Australia, 16: 347-357.

Anderson, J \& J. Ingram. 1994. Tropical soil biology and fertility: a handbook of methods. Soil Science, 157: 1-265.

Barros, E., B. Pashanasi, R. Constantino \& P. Lavelle. 2002. Effects of land use system on the soil macrofauna in western Brazilian Amazonia. Biology and Fertility of Soils, 35: 338-347.

Bohan, D. A., C. W. Boffey, D. R. Brooks, S. J. Clark, A. M. Dewar, L. G. Firbank, A. J. F. Haughton, C. Hawes, M. S. Heard, M. J. May, J. L. Osborne, J. N. Perry, P. Rothery, D. B. Roy, R. J. Scott, G. R. Squire, I. P. Woiwod \& G.T. Champion. 2005. Effects of weed and invertebrate abundance and diversity of herbicide management in genetically modified herbicide-tolerant wintersown oilseed rape. Proceedings of the Royal Society Series B, 272: 463-474.

Bolton, B. 2003. Synopsis and classification of Formicidae. Memoirs of the American Entomological Institute, 71: 1-370.

Bradford, M. A., G. M. Tordof, T. Eggers, T. H. Jones \& J. E. Newington. 2002. Microbiota, fauna, and mesh size interaction in litter decomposition. Oikos, 99: 317-323.

Brown, G., C. Fragoso, I. Barois, P. Rojas, J.C. Patrón, J. Bueno, A.G. Moreno, P. Lavelle, V. Ordaz \& C. Rodríguez. 2001. Diversidad y rol funcional de la macrofauna edáfica en los ecosistemas tropicales mexicanos. Acta Zoológica Mexicana, 1: 79-110.

Brown, G., A. G. Moreno, I. Barois, C. Fragoso, P. Rojas, B. Hernández \& J. C. Patrón. 2004. Soil macrofauna in SE Mexican Pastures and the effect of conversion from native to introduced pastures. Agriculture, Ecosystems and Environment, 103: 313-327. 
Brussard, L. 1998. Soil fauna, functional groups and ecosystem processes. Aplied Soil Ecology, 9: 123-135.

Blüthgen, N., M. Verhaagh, W. Goitía, F. Jaffé, W. Morawetz \& W. Barthlott. 2000. How plants shape the ant community in the Amazonian rainforest canopy: the key role of extrafloral nectaires and homopteran honeydew. Oecologia, 125: 229-240.

Carroll, C. R. \& D. H. Janzen. 1973. Ecology of foraging by ants. Annual Review of Ecology and Systematics, 4: 231-251.

Carroll, C. R. \& S. J. Risch. 1984. The dynamics of seed harvesting in early successional communities by a tropical ant, Solenopsis geminata. Oecologia, 61: 388-392.

Chan, R. 2002. Estudio faunístico de las hormigas (Hymenoptera: Formicidae) de la zona costera entre la localidad de Seybaplaya y Hampolol, Campeche, México. Tesis de Licenciatura. Universidad Autónoma de Campeche. Campeche, México. 61pp.

Chessel, D., A. Dufour \& J.Thioulouse. 2004. The ade4 package-I: One-table methods. $R$ news, 4: 5-10.

CIMAT. 2003. Species Accumulation Function. Freeware. http://cimat.mx/info.php? $\mathrm{m}=1 \&$ ind $=5$

Cokendolpher, J. C. \& O. F. Francke. 1985. Temperature preferences of four species of fire ants (Hymenoptera: Formicidae: Solenopsis). Psyche, 92: 91-102.

Crain, C. \& M. Bertness. 2006. Ecosystem engineering across environmental gradients: Implications for conservation and management. BioScience, 56: 211-218.

Da Silva Moço, M. K., E. F. Da Gama Rodrigues, A. C. Da Gama Rodrigues, R. C. R. Machado \& V. C. Baligar. 2009. Soil and litter fauna of cacao agroforestry systems in Bahia, Brazil. Agroforestry. Systems, 76: 127-138.

Decaëns, T., P. Lavelle, J. J. Jimenez Jaen, G. Escobar \& G. Rippstein. 1994. Impact of land management in the Oriental Llanos of Colombia. European Journal of Soil Biology, 30: 157-168.

Del Toro, I., M. Vázquez, W. P. Mackay, P. Rojas \& R. Zapata Mata. 2009. Hormigas (Hymenoptera: Formicinae) de Tabasco: explorando la diversidad de la mirmecofauna de las selvas tropicales de baja altitud. Dugesiana, 16: 1-14.

Díaz-Francés, E. \& J. Soberón. 2005. Statistical estimation and model selection of species.accumulation functions. Conservation Biology, 19: 569-573.

Dray, S. \& A. B. Dufour. 2007. The ade4 package implementing the duality diagram for ecologist. Journal of Statistical Software, 22: 1-20.

Duffy, J. E. 2002. Biodiversty and ecosystem function: the consumer connection. Oikos, 99: 201-219.

Dufrêne, M. \& P. Legendre. 1997. Species assemblages and indicator species: the need for a flexible asymetrical approach. Ecological Monographs, 67: 345-366.

Evetts, E. A. 2008. Above-and below-ground litter manipulation: effect on retention and release of DOC, DON and DIN in the sikfokut forest, Hungary. Thesis, Texas A\&M University.

Folgarait, P. J. 1998. Ant biodiversity and its relationship to ecosystem functioning: a review. Biodiversity and Conservation, 7: 1221-1244.

Fowler, H. G. 1993. Relative representation of Pheidole (Hymenoptera: Formicidae) in local ground ant assemblages of the Americas. Anales de Biología, 19: 29-37.

González, G. \& T. R. Seatedt. 2001. Soil fauna and plant litter decomposition in tropical and subalpine forest. Ecology, 82: 955-964.

Graham, J. H., A. J. Krzysik, D. A. Kovacic, J. J. Duda, D. C. Freeman, J. M.. Emlen, J. C. Zak, W. Russell Long, M. P. Wallace, C. Chamberlin Graham, J. N. Nutter \& H. E. Balbach. 2008. Ant community composition across a gradient of disturbed military landscapes at Fort Benning, Georgia. Southeastern Naturalist, 7: 429-448. 
Guiseppe, K. F., F. A. Drummond, C. Stubbs \& S. Woods. 2006. The use of Glyphosate herbicides in managed forest ecosystems and their effects on non-target organisms with particular reference to ants as bioindicators. Maine agricultural and forest experiment station the University of Maine, Technical Bulletin, 192 pp.

Gutierrez, J. L. \& C. G. Jones. 2006. Physical ecosystem engineers as agents of biogeochemical heterogeneity. BioScience, 56: 227-236.

Guynn, D. C., S. T. Guynn, T. B. Wigley \& D. A. Miller. 2004. Herbicides and forest diversity—what do we know and where do we go from here? Wildlife Society Bulletin, 32: 1085-1092.

Hooper, D. U., F. S. Chapin, J. J. Ewel, A. Hector, P. Inchausti, S. Lavorel, J. H. Lawton, D. M. Lodge, M. Loreau, S. Naeem, B. Schmid, H. Setälä, A. J. Symstad, J. Vandermeer \& D. A. Wardle. 2005. Ecological Monographs, 75: 3-35.

Huhta, V. 2007. The role of soil fauna in ecosystems: a historical review. Pedobiología (Jena), 50: 489-495.

Jones, C. G., J. H. Lawton \& M. Shachak. 1994. Organism as ecosystem engineers. Oikos, 69: 373386.

Johnson, R. A. \& P. S. Ward. 2002. Biogeography and endemism of ants (Hymenoptera: Formicidae) in Baja California, México: a first overview. Journal of Biogeography, 29: 1009-1026.

Kaspari, M. \& M. D. Weiser. 2000. Ant activity along moisture gradients in a neotropical forest. Biotropica, 32: 703-711.

Kaspari, M., M. N. García, K. E. Harms, M. Santana, S. J. Wrigth \& J. B. Yavitt. 2008. Multiple nutrients limit litterfall and decomposition in a tropical forest. Ecology Letters, 11: 35-43.

Kaspari, M. \& S. P. Yanoviak. 2009. Biogeochemistry and the structure of tropical brown food webs. Ecology, 90: 3342-3351.

Lavelle, P., E. Barros, E. Blanchart, G. Brown, T. Desjardins, L. Mariani \& R. Jean-Pierre. 2001. SOM management in the tropics: Why feeding the soil macrofauna? Nutrient Cycling in Agroecosystems, 61: 53-61.

Lavelle, P., B. Senapati \& E. Barros. 2003. Soil macrofauna. Pp. 303-324. In: G. Schroth \& F. L. Sinclair (Eds.). Trees, Crops and Soil Fertility. CABI Publishing.

Levings, S. C. 1983. Seasonal, annual, and among-site variation in the ground ant community of a deciduos tropical forest: Some causes of patchy species distribution. Ecological Monographs, 53: 435-455.

Lieberburg, I., P. M. Kranz \& A. Seip. 1975. Bermudian ants revisited: the status and interactions of Pheidole megacephala and Irydomyrmex humilis. Ecology, 56: 473-478.

Lighton, J. R. B. \& D. H. Feener. 1989. Water loss rate and cuticular permeability in foragers of the desert ant Pogonomyrmex rugosus. Physiological Zoology, 62: 1232-1256.

Lok, R. 1998. Introducción a los huertos caseros tradicionales tropicales. CATIE-GTZ. Turrialba, Costa Rica. 157 pp.

Masera, O., M. Astier \& S. López-Ridaura. 1999. Sustentabilidad y manejo de recursos naturales. El marco de evaluación MESMIS. Mundi Prensa México, SA de CV, México D.F., México. 109 pp.

MacGown, J. A. \& T. T. Lockley. 2009. Ants of Horn Island, Jackson County, Mississippi. In: Ants (Formicidae) of the Southeastern United States. MacGown, J.A. Mississippi Entomological Museum. http://mississippientomologicalmuseum.org.msstate.edu/Researchtaxapages/Formicidaepages/faunal.lists/HornIsland.Ants.htm

McGeoch, M. A., B. J. Van Rensburg \& A. Botes. 2002. The verification and application of bioindicators a case study of duna beetles in a savanna ecosystem. Journal of Applied Ecology, 39: 661-672. 
Montagnini, F. 2006. Homegardens of Mesoamérica: Biodiversity, food security, and nutrient management. pp.61-86. In: Kumar, B.M. \& Nair, P.K.R. (Eds.). Tropical Homegardens: A time - tested example of sustainable agroforestry. Springer, Dordrecht, Holanda.

Orellana, L.R. 1999. Evaluación Climática. Pp. 163-182. In: P.A. Chico (Ed.). Atlas de procesos territoriales de Yucatán. Universidad Autónoma de Yucatán, Facultad de Arquitectura.

Peck, S. I., B. Mcquaid \& C. L. Campbell. 1998. Using ant species as a biological indicator of Agroecosystem condition. Environmental Entomology, 27: 1102-1110.

Perfecto, I. 1994. Foraging behavior as a determinant of asymmetric competitive interactions between two ant species in a tropical agroecosystem. Oecologia, 98: 184-192.

Perfecto, I \& A. Sediles. 1992. Vegetational diversity, ants (Hymenoptera, Formicidae), and herbivorous pests in a neotropical agroecosystem. Environmental. Entomology, 21: 61-67.

Perfecto, I. \& J. Vandermeer. 1996. Microclimate changes and the indirect loss of ant diversity in a tropical agroecosystems. Oecologia, 108: 577-582.

Peterson, R.K.D. \& A.G. Hulting. 2004. A comparative ecological risk assessment for herbicides used on spring wheat: the effect of glyphosate when used within a glyphosate-tolerant wheat system. Weed Science, 52: 834-844.

Philpott, S. M. \& I. Armbrecht. 2006. Biodiversity in tropical agroforest and the ecological role of ants and diversity in predatory function. Ecological Entomology, 31: 369-377.

Potts, L. R., J. C. Cokendolpher \& O. F. Francke. 1983. Humidity preferences of four species of fire ants (Hymenoptera: Formicinae: Solenopsis). Insectes Sociaux, 31: 335-339.

Quiroz-Robledo, L. N. \& J. Valenzuela González. 2007. Distribution of Poneromorph ants (Hymenoptera:Formicidae) in the Mexican state of Morelos. Florida Entomologist, 90: 609-615.

R. Development Core Team. 2008. R: A language and environment for statistical computing. R Foundation for Statistical Computing, Vienna, Austria. http://www.R-project.org.

Risch, S. 1980. Ants as important predators of rootworm eggs in the neotropics. Journal of Economic Entomology, 74: 88-90.

Risch, S. J. \& C. R. Carroll. 1982. The ecological role of ants in two Mexican agroecosystems. Oecologia, 55: 114-119.

Rivas C. R. \& J. H. Schoereder. 2007. Ant communities, environmental characteristics and their implications for conservation in the Brazil Pantanal. Biodiversity and Conservation, 16: 1511-1520.

Rojas, P. 2001. Las hormigas del suelo en Mexico: diversidad, distribucion e importancia (Hymenoptera: Formicidae). Acta Zoologica Mexicana, 2001 (no. especial 1): 189-238.

Römbke, J. \& M. García. 2000. Assessment of Ecotoxicological effects of pesticides on the soil fauna and soil processes under tropical conditions. German-Brazilian Workshop on Neotropical Ecosystems - Achievements and Prospects of Cooperative Research. Hamburg, Germany. 7 pp.

Sayer, E. J. 2006. Using experimental manipulation to assess the roles of leaf litter in the functioning of forest ecosystems. Biological Reviews, 81: 1-31.

Ter-Braak, C. 1986. Canonical correspondence analysis: A new eigenvector technique for multivariate direct gradient analysis, Ecology, 67: 1167-1179.

Torquebiau, E. \& E. Penot. 2006. Ecology versus economics in tropical multistrata agroforests. pp. 269-282. In: B. M. Kumar \& P. K. R. Nair (Eds.). Tropical Homegardens: A time-tested example of sustainable agroforestry. Springer, Dordrecht, Holanda.

Torres-Contreras, H. \& R. A. Vásquez. 2004. A field experiment on the influence of load transportation and match distance on the locomotion velocity of Dorymyrmex goetschi (Hymenoptera, Formicidae). Insectes Sociaux, 51: 265-270. 
Van Rensburg, B. J., M.A. McGeoch, S. L. Chown \& A. S. Van Jaarsveld. 1999. Conservation of heterogeneity among duna beetles in the Maputaland Centre of Endemism, South Africa. Biological Conservation, 88: 145-153.

Vasconcelos, H. L. \& W. F. Laurance. 2005. Influence of habitat, litter type, and soil invertebrates on leaf-litter decomposition in a fragmented Amazonian landscape. Oecología, 144: 456-462.

Wilson, E. O. 1978. Division of labor in fire ants based on physical castes (Hymenoptera: Formicidae: Solenopsis). Journal of the Kansas Entomological Society, 51: 615-636.

Wolters, V., W. L. Silver, D. C. Bignell, D. C. Coleman, P. Lavelle, W. Van der Putten, P. De Ruiter, J. Rusek, D. H. Wall, D.A. Wardle, L. Brussaard, J. M. Dangerfield, V.K. Brown, K. E. Giller, D.U. Hooper, O. Sala, J. Tiedje \& J.A. Van Veen. 2000. Effects of global changes on above and belowground biodiversity in terrestrial ecosystems: implications for ecosystem functioning. BioScience, 50: 1089-1098. 